\title{
Sterility and Delayed Vaginal Opening Following Electrolytic Lesions Placed Neonatally in the Medial Preoptic Area in Female Rats
}

\author{
SHINJI HAYASHI AND TOMOKo ORIMO \\ Endocrinology Division, National Cancer Center Research Institute, \\ Tsukiji 5-chome, Chuo-ku, Tokyo 104
}

\begin{abstract}
Synopsis
Electrolytic lesions were placed in the preoptic area or in the middle and posterior hypothalamus of female rats within $48 \mathrm{hrs}$ after birth. Vaginal opening was delayed significantly in rats having the medial preoptic area (mPOA) destructed as compared to sham-operated animals and those given lesions in the other parts of the hypothalamus. Only rats with the mPOA completely destructed developed the anovulatory syndrome. In these animals, unilateral ovariectomy was not followed by enlargement of its fellow. The present results are not in agreement with those of previous workers that electrolytic destructions of the diencephalon induced precocious puberty. The difference may be ascribable to difference in size and locus of lesions.
\end{abstract}

It is well known that the anterior hypothalamus or the areas anterior to the hypothalamus of the brain in female rats are responsible for sexual cyclicity or ovulatory discharge of gonadotropins from the anterior pituitary. Placement of electrolytic lesions in some hypothalamic areas of adult female rats induces a syndrome characterized by polyfollicular, anovulatory ovaries and persistent vaginal cornification (Greer, 1953; D'Angelo and Kravatz, 1960 ; Taleisnik and McCann, 1961). A similar syndrome also occurs in adult rats following anterior neuronal deafferentation of the hypothalamus (Halász and Gorski, 1967) and in those having been administered with either androgens or estrogens during neonatal life (For reviews, Takewaki, 1962; Gorski, 1971; Arai, 1973). Androgen micropellets placed in the ventromedial-arcuate nucleus region of the hypothalamus (Nadler, 1972)

Received for publication March 31, 1975. or within a rather wide, undefinable area of the basal hypothalamus (Wagner et al., 1966; Hayashi and Gorski, 1974) of neonatal female rats were shown to induce anovulatory sterility in adulthood. Barraclough and Gorski (1961) suggested that the site affected by neonatal androgen injection in the hypothalamus is the site destruction of which results in the anovulatory syndrome.

On the other hand, several workers have reported that, if the anterior hypothalamus is destructed in female rats at 3-4 days of age (Horowitz and van der Werff ten Bosch, 1962; Relkin, 1968) or at 22 or 24 days (Gellert and Ganong, 1960; Meijs-Roelofs and Moll, 1972), precocious vaginal opening indicative of the onset of puberty takes place. The onset of puberty is also advanced in female rats by hypothalamic deafferentation (Ramaley and Gorski, 1967).

The experiments reported here indicate that destruction of the medial preoptic area 
(mPOA) causes a delay of vaginal opening and may bring about an anovulatory syndrome.

\section{Materials and Methods}

In 14 2-day-old female rats of the Sprague-Dawley strain, bilateral electrolytic lesions $(1 \mathrm{~mA}$, D.C. $\times 30$ sec) were placed in the antero-basal part of the forebrain ('anterior' lesions aimed at the preoptic to anterior hypothalamic areas) by means of a monopolar electrode (anodal, stainless steel electrode, 0.4 $\mathrm{mm}$ in diameter) and in 14 other females, lesions were placed in the middle to posterior hypothalamus ('posterior' lesions aimed at the area between the ventromedial hypothalamus and the mamillary bodies) by using similar electrode. A neutral electrode was placed on the skin incision. In 9 sham-operated rats the electrode was inserted into the same areas of the brain ( 5 anterior and 4 posterior) but the current was not passed. The stereotaxic procedures were the same as those described previously (Hayashi, 1974). Four intact litter-mates of those given electrolytic lesions served as controls. The operated pups were returned to their mothers immediately after regaining conciousness with whom they remained until they were weaned at 28 days of age. The day of vaginal opening was recorded in each animal and daily vaginal smears were followed. Left ovaries were removed at 60 days of age and weighed regardless of the stage of estrous cycle. Exactly 2 weeks later, the animals were sacrificed, right ovaries, adrenals, thyroids and uteri were taken out, weighed and fixed in Bouin's solution. The materials were sectioned at $10 \mu$ in paraffin and stained with Delafield's hematoxylin and eosin. Brains were removed after perfusion with a mixture of equal volumes of $1 \%$ potassium ferro- and ferri-cyanide solutions followed by a buffered $10 \%$ formalin, imbedded in gelatin, sectioned while frozen and stained with carbol fuchsin. Loci of brain lesions were determined microscopically. Animals without corpora lutea (CL) in their ovaries were classified as sterile. Significance of differences was tested with Student's $t$ test.

\section{Results}

Histological examination of the brains revealed that in 4 out of 14 female rats which had been given the 'anterior' lesions in the basal part of the forebrain, the mPOA was almost completely destroyed on both sides (Group $A_{1}$, Fig. 1, crosshatched areas). Considerable damage was occasionally caused to the septal area, anterior commissure (CA), stria terminalis and diagonal band (DBB). One of the 4 rats showed persistent-estrous and 2 other animals persistent-diestrous vaginal smears. In the 4th animal, vaginal orifice was not formed until the day of sacrifice (74 days of age). Neither ovaries from the same rats contained CL. Their ovarian weight was significantly smaller than those from the other groups of rats (Table 1). Ovarian compensatory hypertrophy $(\mathrm{OCH})$ was less marked and the weights of the body and uterus were also significantly smaller in Group $A_{1}$ rats compared to those of shamcontrol animals (Tables 1 and 2). The adrenal and thyroidal weights were not significantly different among groups as expressed on a $\mathrm{mg} / 100 \mathrm{~g}$ body weight basis (Table 2).

In the remaining 10 females bearing 'anterior' lesions (Group $A_{2}$ ), the electrolytic lesions were relatively small and diffuse,

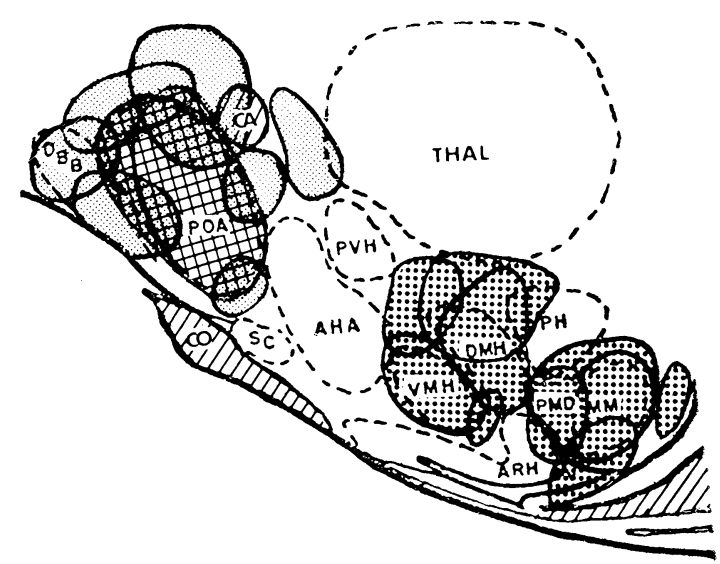

Fig. 1. Sagittal section of basal forebrain illustrating locations of lesions. Destructed areas in Group $A_{1}$ rats are crosshatched, those in Group $A_{2}$ rats finely dotted, and those in Group $\mathrm{P}$ rats coarsely dotted. Abbreviations: ARH, arcuate nucleus; $\mathrm{CO}$, optic chiasm; $\mathrm{PH}$, posterior hypothalamic nucleus; POA, preoptic area; $\mathrm{PVH}$, paraventricular nucleus; SC, suprachiasmatic nucleus and THAL, thalamus. For others, see text. 
located in and around the mPOA (e.g. in $\mathrm{DBB}$, septum, CA, lateral parolfactoral area and anterior hypothalamic area (AHA)) but the mPOA was not completely destructed (Fig. 1, finely dotted areas). The estrous cycles of these animals were irregular, their ovaries always bearing some CL.

Vaginal opening was delayed in the 13 rats bearing 'anterior' lesions (Groups $\mathrm{A}_{1}$ and $\mathrm{A}_{2}, 46.6 \pm 1.6$ days of age) compared to that in sham-controls $(40.3 \pm 1.1$ days, $\mathrm{p}<0.01)$.

On the other hand, in the 14 female rats with 'posterior' lesions (Group P) electrolytic lesions were relatively small and located in or around the ventromedial hypothalamic (VMH), dorsomedial hypothalamic (DMH), dorsal premamillary (PMD) and medial mamillary (MM) nuclei (Fig. 1, coarsely dotted areas). The estrous cycles were irregular in 10 of these rats and regular in the remaining 4. All the rats had CL in their ovaries. No difference was present in age of vaginal opening, ovarian weights and $\mathrm{OCH}$ compared to those from the sham-controls (Table 1).

\section{Discussion}

Destruction or severance of neuronal pathways in the rostral hypothalamus in adult female rats is reported to inhibit the ovulatory discharge of gonadotropins, result-

Table 1. Mean age in days of vaginal opening (M. \pm S.E.) and ovarian weights (M. \pm S.E.) in rats given lesions in different parts of the forebrain at 2 days of age. The medial preoptic area was destroyed completely in Group $A_{1}$ and partially in Group $A_{2}$. In Group $\mathbf{P}$ animals the area ranging from the ventromedial to the mamillary nuclei was destructed.

\begin{tabular}{|c|c|c|c|c|c|c|c|}
\hline \multirow[b]{2}{*}{$\begin{array}{l}\text { Experimental } \\
\text { groups }^{\mathrm{a}}\end{array}$} & \multirow[b]{2}{*}{$\begin{array}{l}\text { No. of } \\
\text { treated rats }\end{array}$} & \multirow[b]{2}{*}{$\begin{array}{l}\text { No. of } \\
\text { sterile rats }\end{array}$} & \multirow[b]{2}{*}{$\begin{array}{l}\text { IS }^{\mathbf{b}} \\
(\%)\end{array}$} & \multirow{2}{*}{$\begin{array}{c}\text { Vaginal } \\
\text { opening } \\
\text { (day) }\end{array}$} & \multicolumn{2}{|c|}{ Ovarian weights } & \multirow[b]{2}{*}{$\begin{array}{c}\mathrm{OCH}^{\mathrm{c}} \\
(\%)\end{array}$} \\
\hline & & & & & $\begin{array}{c}\text { Left } \\
(\mathrm{mg} / 100 \mathrm{~g}\end{array}$ & $\begin{array}{c}\text { Right } \\
\text { body weight) }\end{array}$ & \\
\hline Intact-control & 4 & 0 & 0 & $36.8 \pm 2.2$ & $16.5 \pm 0.7$ & $30.2 \pm 1.8$ & $103 \pm 10$ \\
\hline Sham-control & 9 & 0 & 0 & $40.3 \pm 1.1$ & $17.9 \pm 0.9$ & $28.2 \pm 1.5$ & $83 \pm 7$ \\
\hline $\mathrm{A}_{1}$ & 4 & 4 & 100 & $45.0^{\mathrm{d}}$ & $7.1 \pm 0.8^{* *}$ & $7.8 \pm 1.2 * *$ & $25 \pm 5^{* *}$ \\
\hline $\mathrm{A}_{2}$ & 10 & 0 & 0 & $47.1 \pm 2.0 *$ & $15.0 \pm 1.3$ & $24.7 \pm 1.7$ & $104 \pm 15$ \\
\hline $\mathbf{P}$ & 14 & 0 & 0 & $40.4 \pm 1.3$ & $17.0 \pm 1.2$ & $28.6 \pm 2.0$ & $98 \pm 12$ \\
\hline
\end{tabular}

a : For explanation, see text.

b : Incidence of sterility (IS)

$$
=\frac{\text { No. of female rats without corpora lutea }}{\text { no. of treated rats }} \times 100 \%
$$

c : Ovarian compensatory hypertrophy $(\mathrm{OCH})$ $=\frac{\text { Right ovarian weight }- \text { left ovarian weight }}{\text { left ovarian weight }} \times 100 \%$

d : Data from 3 of 4 rats, the remaining one did not show vaginal opening by 74 days of age.

$*$ and **: Significantly different from sham-controls $(\mathrm{p}<0.01$ and $<0.001$, respectively).

Table 2. Body and organ weights (Mean \pm S.E.) of different groups of rats (Table 1).

\begin{tabular}{lccccc}
\hline \hline $\begin{array}{c}\text { Experimental } \\
\text { groups }\end{array}$ & $\begin{array}{c}\text { No. of } \\
\text { rats }\end{array}$ & $\begin{array}{c}\text { Body weight } \\
(\mathrm{g})\end{array}$ & Thyroids & $\begin{array}{c}\text { Adrenals } \\
(\mathrm{mg} / 100 \mathrm{~g} \text { body weight })\end{array}$ & Uterus \\
\hline Intact-control & 4 & $227 \pm 7$ & $7.9 \pm 0.4$ & $32.4 \pm 2.3$ & $233 \pm 11$ \\
Sham-control & 9 & $228 \pm 8$ & $8.4 \pm 0.6$ & $30.9 \pm 2.4$ & $221 \pm 12$ \\
$\mathrm{~A}_{1}$ & 4 & $163 \pm 9 *$ & $9.3 \pm 0.4$ & $26.8 \pm 2.7$ & $95 \pm 29^{* *}$ \\
$\mathrm{~A}_{2}$ & 10 & $216 \pm 6$ & $8.5 \pm 0.3$ & $31.2 \pm 1.5$ & $175 \pm 7^{*}$ \\
$\mathrm{P}$ & 14 & $201 \pm 13$ & $8.7 \pm 0.5$ & $32.5 \pm 0.9$ & $218 \pm 14$ \\
\hline
\end{tabular}

* and **: Significantly different from values of sham-control $(\mathrm{p}<0.01$ and $<0.001$, respectively). 
ing in anovulation and persistent vaginal estrus or diestrus (Greer, 1953; D'Angelo and Kravatz, 1960; Taleisnik and McCann, 1961; Halász and Gorski, 1967). Barraclough and Gorski (1961) suggested that the neuronal components responsible for regulating the phasic secretion of gonadotropins are located in the preoptic-suprachiasmatic area.

The present findings indicate that the preoptic area and rostral hypothalamus has already been determined to regulate the ovulatory discharge of gonadotropins after maturity by $48 \mathrm{hrs}$ after birth, since the complete destruction of the area (mPOA) in neonates resulted in the anovulatory syndrome in adulthood. The effects of the partial destruction of the area were less marked, only a reduction of the uterine weight and irregular sexual cycles being the outcome. When the mPOA was partially destructed, the remains of the area might effectively control the ovulatory gonadotropin-discharge in adulthood. In this context, it is suggested that the total destruction of mPOA might block the neuronal influences involved in the regulation of the ovulatory discharge of gonadotropins.

The fact that compensatory hypertrophy was rarely observed in remaining ovaries of Group $A_{1}$ rats after hemiovariectomy may indicate that the extensive destruction of mPOA have also damaged systems which are involved in gonadotropin rise after hemiovariectomy.

In the present experiments, care was taken not to destruct the stalk-median eminence region. The absence of any significant difference both in adrenal and thyroidal weights among the different experimental groups might be ascribable to the intactness of the stalk-median eminence region. Since many of the animals bearing brain lesions in the posterior as well as the anterior hypothalamus showed irregular vaginal cycles, it seems that the nuclei in these regions are also involved in the oc- currence of regular sexual cycles.

Nadler (1972) reported that the placement of androgen crystals in the ventromedial-arcuate region of neonatal female rats resulted in anovulatory sterility, while similar crystals located in the POA were without effect in this respect. However, the present results seem to indicate that the site responsible for the mechanism of the ovulatory release of gonadotropins is located in the mPOA.

Several workers have reported that precocious puberty might be induced by placement of lesions in the hypothalamus. According to Elwers and Critchlow (1960), electrocauterization of the anterior hypothalamus in female rats at 18-20 days of age was followed by precocious puberty. Horowitz and van der Werff ten Bosch (1962) and Relkin (1968) also hastened the onset of puberty by placing lesions in the rostral hypothalamus in 3-4 days old female rats. On the contrary, Gellert and Ganong (1960) and Bloch and Ganong (1971) advanced the arrival of puberty in female rats by destroying the posterior tuberal region anterior to the mamillary bodies but not by destructing the anterior hypothalamus. Meijs-Roelofs and Moll (1972) suggested that the size of lesions placed in the hypothalamus is of primary importance in the induction of precocious puberty, since vaginal opening is likely hastened by influences from the region dorsal to the arcuate nucleus but inhibited by influences from the other parts of the hypothalamus. However, in the present experiments, delayed vaginal opening was induced by destructing the anterior hypothalamus, POA and some parts of the anterior limbic forebrain, while cauterization in the posterior hypothalamus had no effect. The delay of vaginal opening is unlikely due to the ratardation of body growth as found in Group $A_{1}$ rats, since body weights of Group $\mathrm{A}_{2}$ rats were almost same as those of controls (Table 2). Our findings are not in agreement with 
those reported by Horowitz and van der Werff ten Bosch (1962) and Relkin (1968) that vaginae opened precociously following destruction of the retrochiasmatic region in female rats at 3-4 days of age. The discrepancies between the findings of the previous workers and ours might be due to difference in size and locus of destructed areas. The previous workers placed lesions mainly in the suprachiasmatic nucleus region, whereas in the present experiments effective lesions were located more anterior part of the forebrain. However, further studies are needed to clarify the relationship between the loci of lesions and the time of vaginal canalization.

\section{Acknowledgements}

The authors are indebted to Prof. K. Takewaki of Kawasaki Medical College for his valuable criticism and help in preparing the manuscript. This work was partly supported by a research grant from the Japanese Ministry of Health and Welfare.

\section{References}

Arai, Y. Neuroendocrine Control (edited by $\mathrm{K}$. Yagi and S. Yoshida). Univ. Tokyo Press, Tokyo, p. 27 (1973).

Barraclough, C. A. and R. A. Gorski (1961). Endocrinology 68, 68.

Bloch, G. J. and W. F. Ganong (1971).
Ibid. 89, 898.

D'Angelo, S. A. and A. S. Kravatz (1960). Proc. Soc. Exp. Biol. Med. 104, 130.

Elwers, M. and V. Critchlow (1960). Am. J. Physiol. 198, 381.

Gellert, R. J. and W. F. Ganong (1960). Acta Endocr. (Kbh) 33, 569.

Gorski, R. A. Frontiers in Neuroendocrinology, (edited by L. Martini and W. F. Ganong). Oxford Univ. Press, N. Y., p. 237 (1971).

Greer, M. A. (1953). Endocrinology 53, 380.

Halász, B. and R. A. Gorski (1967). Ibid. 80, 608 .

Hayashi, S. (1974). Endocrinol. Japon. 21, 453.

Hayashi, S. and R. A. Gorski (1974). Endocrinology 94, 1161.

Horowitz, S. and J. J. van der Werff ten Bosch (1962). Acta Endocr. (Kbh) 41, 301.

Meijs-Roelofs, H. M. A. and J. Moll (1972). Neuroendocrinology 9, 297.

Nadler, R. D. (1972). Ibid. 9, 349.

Ramaley, J. A. and R. A. Gorski (1967). Acta Endocr. (Kbh) 56, 661.

Relkin, R. (1968). Endocrinology 82, 865.

Takewaki, K. (1962). Experientia 18, 1.

Taleisnik, S. and S. M. McCann (1961). Endocinology 68, 263.

Wagner, J. W., W. Erwin and V. Critchlow (1966). Ibid. 79, 1135. 